\section{The megalab truth test}

SIR - Results from a new opinion.poll suggest that lying in everyday life is more widespread than had previously been assumed $^{1}$. Only $12 \%$ of the sample claimed that they had never told a lie, whereas $24 \%$ claimed to have lied at least once during the previous day. It is thus worrying that a large body of research indicates that most individuals are unable reliably to detect when others are lying ${ }^{2}$.

Much of this work has concentrated on identifying the cues used by observers to decide whether another individual is

\begin{tabular}{llll}
\hline \multicolumn{3}{c}{ MEGALAB TRUTH TEST RESULTS } \\
\hline & Newspaper & Radio & Television \\
No. correct & 2,207 & 769 & 19,165 \\
No. incorrect & 1,231 & 279 & 17,820 \\
$\%$ correct & 64.2 & 73.4 & 51.8 \\
$\chi^{2}$ value & 277 & 229 & 49 \\
$\quad$ (d.f. = 1) & & & \\
$P$ value & $<1 \times 10-14$ & $<1 \times 10-14$ & $<1 \times 10-14$ \\
\hline
\end{tabular}

All $\chi^{2}$ and $P$ values calculated by testing actual distributions against a theoretical 50/50 chance distribution.

telling the truth ${ }^{3,4}$. Such cues fall into three broad categories: verbal cues, consisting of just the words used by the liar (including the number of words spoken, length of sentences); vocal cues, involving the way in which these words are said (voice pitch, pauses, hesitation); and visual cues, including any observable signs given off during the communication (eye contact, body movement, facial expressions). In a typical study, individuals are presented with films of liars and truth-tellers (containing all three types of cue), soundtracks of these films (containing vocal and verbal cues) or just the films' written transcripts (containing only verbal cues). The results have tended to be counterintuitive, with individuals watching the films (and therefore receiving the largest number of cues) exhibiting the lowest detection rates.

Many of these studies have been carried out with relatively small numbers of individuals (mostly university students) in laboratory environments. As a result, their conclusions may be applicable only to this rather limited set of circumstances. Despite the obvious importance of these results, no previous work had examined whether such findings generalize to a much wider cross-section of the population using actual newspapers, radio and television. Our 'megalab truth test' was designed to resolve this issue.

A well known British political commentator (Sir Robin Day) was interviewed twice about his favourite films. In one interview he consistently told the truth, in the other he consistently lied. Transcripts of these interviews were printed in The Daily Telegraph, broadcast on BBC Radio 1 and shown on the BBC Tomorrow's World television programme. People were asked to decide which interview contained the lies and to telephone appropriate numbers to record their decision.

There was a huge response from the public $(n=41,471)$. Radio listeners detected the lies $73.4 \%$ of the time, newspaper readers $64.2 \%$, and television viewers $51.8 \%$. All three groups could detect the lies at above-chance levels (see table). Perhaps more interesting, there were significant differences between the detection rates of the three groups (d.f. $=2$, $\left.\chi^{2}=366, \quad P<1 \times 10^{-14}\right)$. These differences support the notion that the presence of visual cues reduces individuals' ability to detect deception.

Clearly, the study was not perfect. The judgements were based on two relatively short interviews with just one person. The original design was to interview several politicians but this had to be abandoned due to the limited amount of broadcast time. Second, it was not possible randomly to allocate individuals to the three conditions. Third, one has to assume that the technology used to collect and collate a large number of responses in a short time was accurate and reliable. But despite these problems the study produced striking results and it is difficult to conclude that they are not due, at least to some extent, to the effect of the three media.

These results have important implications. Several studies have now shown that vocal and verbal cues tend to be more reliable indicators of deceit than visual cues ${ }^{1}$. Despite this, observers watching videotapes of potential liars seem to base their decisions on visual cues. This may be because visual cues are more compelling than verbal/vocal ones or because observers falsely believe that such signals (for example, eye contact) are the best indicator of deceit. Whatever the explanation, it is clear that individuals wishing to detect deceit might be better off consciously paying more attention to verbal or vocal, as opposed to visual, cues.

The truth test was designed, in part, to increase public understanding of science. Although not perfect, the experiment illustrated how certain experimental methods (for example comparing performance between three conditions, 'blind' judging and so on) could be used to investigate an interesting and important issue.
In addition, the experiment received a large amount of media coverage ${ }^{5}$ and generated greater public involvement. Perhaps most important of all, it yielded interesting and useful data. That is assuming, of course, that the public were telling us the truth.

\section{Richard Wiseman}

Division of Psychology,

University of Hertfordshire,

College Lane,

Hatfield,AL10 9AB, UK

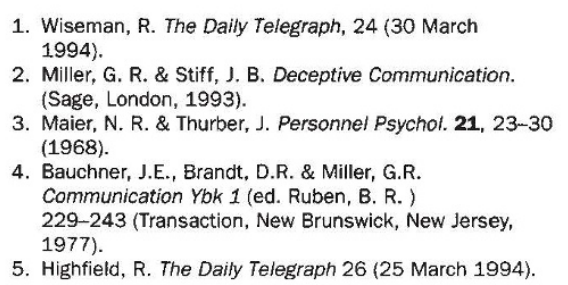

\section{Spores in earliest land plants}

SIR - The earliest evidence for the colonization of the land surface by plants is in the form of desiccation-resistant spores ${ }^{1}$. These spores predate taxonomically recognizable macrofossils by 30 million years. The material that makes up the walls (or exospores) of these spores sporopollenin - maintains its fine structure over long spans of geological time. It is possible to document the ultrastructure of these dispersed spores using the electron microscope, and to infer relationships to modern plants by comparing their spore-wall ultrastructure.

I examined members of the dispersed spore genus Dyadospora (Lower Silurian Centerville Formation, Adams County, Ohio; Fig. 1). A cross-section of the exospore reveals a two-part construction (Fig. 2). An inner region, $1.4 \mu \mathrm{m}$ thick when not deformed, is constructed of 10-15 distinct plate-shaped units or lamellae, each 50-150 nm thick. The outer region is $700-1,500 \mathrm{~nm}$ thick, and is not obviously lamellated.

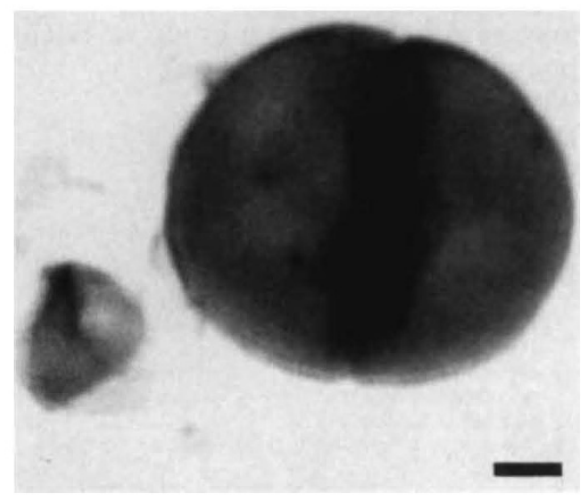

FIG. 1 Dyadospora sp. Light micrograph showing two adjacent spores of this dyad. Scale bar, $10 \mu \mathrm{m}$. 